\title{
Reduced Solar Activity Disguises Global Temperature Rise
}

\author{
Peter Stauning \\ Danish Meteorological Institute, Copenhagen, Denmark \\ Email:pst@dmi.dk
}

Received November 7, 2013; revised November 30, 2013; accepted December 8, 2013

Copyright (C) 2014 Peter Stauning. This is an open access article distributed under the Creative Commons Attribution License, which permits unrestricted use, distribution, and reproduction in any medium, provided the original work is properly cited. In accordance of the Creative Commons Attribution License all Copyrights (C) 2014 are reserved for SCIRP and the owner of the intellectual property Peter Stauning. All Copyright (C) 2014 are guarded by law and by SCIRP as a guardian.

\begin{abstract}
The question whether human activities seriously affect climate is asked with increasing voice these days. Quite understandable since the climate appears to be out of control with the significant global temperature increases already seen during the last three decades and with still heavier temperature increases to come in the future according to prognoses, among others, in the recent comprehensive IPCC reports [1]. However, the most recent climate data [2], show global temperature development levelling off or even turning negative since 2001 in contrast to the anticipated course related to the steady increases in the concentration in the atmosphere of green-house gasses, primarily carbon dioxide and methane [1]. The purpose of this communication is to demonstrate that the reduced rate in the global temperature rise complies with expectations related to the decaying level of solar activity according to the relation published in an earlier analysis [3]. Without the reduction in the solar activity-related contributions the global temperatures would have increased steadily from 1980 to present.
\end{abstract}

\section{KEYWORDS}

Solar Activity; Earth's Climate; Global Temperatures

\section{Introduction}

The alarming rise in global temperatures from about 1980 to 2000 gave much concern around possible serious future climate changes, global warming, that could result from the increasing levels of carbon dioxide, methane and other greenhouse gasses in our atmosphere. However, as shown in [2] the strong rise in global temperatures faded after year 2000 and was replaced by a rather steady level or even small decreases in the global temperatures from around 2001 to present (2013). This development took away some of the incitement to cut down on human-induced growth in greenhouse gasses.

The question is now whether the present fading of the temperature rise is related to the concurrent decrease in solar activity scaled, for instance, by the sunspot numbers. Scientists have linked past climate changes to solar activity. The so-called “Little Ice Age” in the 17'th century was linked to the Maunder minimum in solar activity by [4]. Many later works have linked climate changes to changes in solar activity (see reviews [5,6]).

In the earlier analysis [3] from the Danish Meteoro- logical Institute (DMI) a quantitative assessment was made of the relation between solar activity represented by the cycle-average sunspot numbers and the terrestrial climate represented by the global temperatures averaged over the same interval length but delayed by 3 years. In the present communication the anticipated effects of the developments in solar activity on the recent global temperature changes are analyzed.

\section{Sunspots and Global Temperatures}

The former analysis [3] and the present work assume that solar activity can be represented through the classical international sunspot number SSN $=\mathrm{k} \cdot(\mathrm{s}+10 \cdot \mathrm{g})$, where $\mathrm{s}$ is the number of sunspots, $g$ the number of sunspot groups while $\mathrm{k}$ is a calibration parameter to ensure that different observatories derive the same sunspot number regardless of observational qualities. A discussion of this index and of modified versions of the sunspot number is provided by [7]. The sunspot number is used here rather than satellite-based observations of solar radiation because of the extended length of the time interval of 
available data.

The international sunspot numbers are explained and made available from the Solar Influences Data Analysis Center (SIDC) in Belgium, among other, through their web site [8]. The SIDC observed and smoothed sunspot numbers for the ongoing cycle 24 up to present are shown in Figure 1. For the sunspot values beyond present, according to [9], predicting the behavior of a sunspot cycle is fairly reliable once the cycle is well underway (about 3 years after the minimum in sunspot number). Presently (2013) we are about 4 years into cycle 24 .

Figure 1 also displays the extensions through 1.5 years derived at SIDC with different models (kfsm "classical standard" and kfcm "combined” models). The figure, furthermore, displays the predictions prepared by the Australian IPS Radio and Space Services [10] and the NASA solar cycle 24 predictions [11] as of October 2013.

The mean of the two SIDC extrapolations [8] 1.5 years ahead as well as the NASA prediction places the maximum of cycle 24 in mid-2013. The currently observed and predicted sunspot numbers makes this sunspot cycle the weakest since cycle 14 which had a maximum in the smoothed data of 64.2 in February of 1906. When final sunspot data become available they may turn out still lower to make cycle 24 even weaker than cycle 14.

Sunspot numbers have been reconstructed back to around 1850 with quite good accuracy based on astronomers' careful and detailed recordings of the appearance of the solar surface. The yearly sunspot numbers since 1850 available from SIDC [8] are shown by the thin blue line in the bottom panel of Figure 2. The extension shown by the dashed line from present through the remaining solar cycle 24 to 2020 is based on the mean of the IPS [10] and the NASA [11] predictions.

The bottom panel of Figure 2 also displays the averages of sunspot number from minimum to minimum

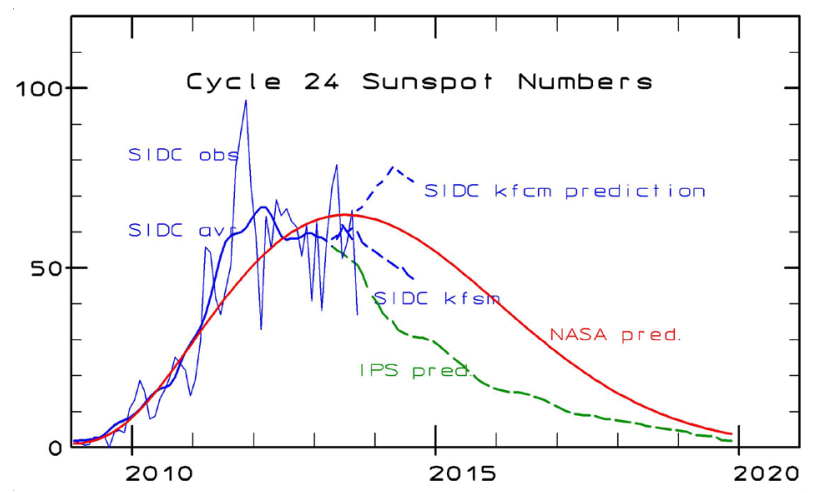

Figure 1. Sunspot numbers. From SIDC: observed (thin blue line), smoothed (heavy blue line), kfsm predictions (blue dashed line), and $\mathbf{k f c m}$ predictions (blue dotted line). IPS predicted extensions (green dashed line), and NASA predictions (red line).

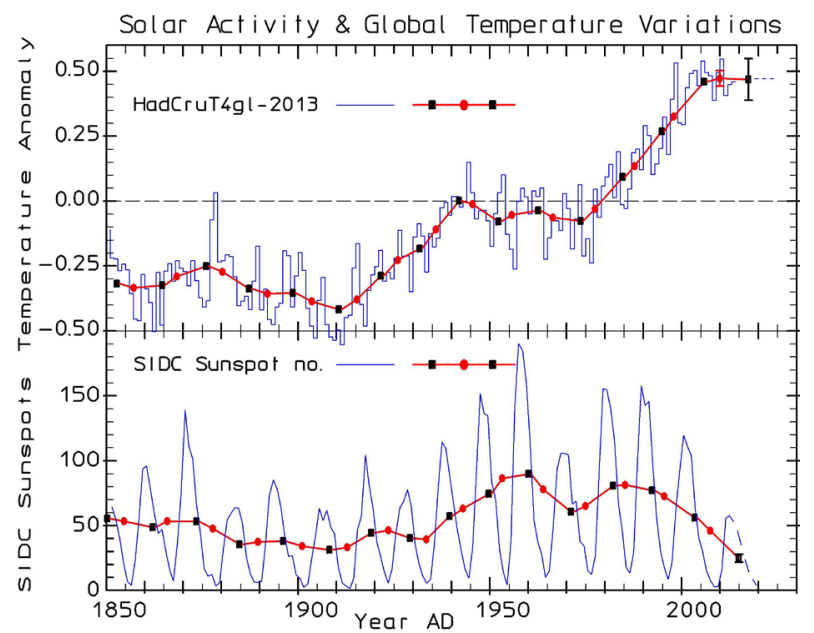

Figure 2. Upper panel: HadCRUT-4gl series (2013) for combined land-surface/sea-surface yearly and cycle average global temperatures. Bottom panel: SIDC sunspot numbers and cycle-average sunspot numbers (heavy line with dots).

(usual solar cycle) marked by squares and from maximum in a cycle to maximum in the next cycle marked by filled circles.

The marks are connected by the heavy red line. The averaging is made this way to provide a fair representation of solar activity regardless of the strongly varying solar cycle length (from 9 to 15 years). Except for the present cycle 24, the sunspot minima and maxima years are derived from the NOAA-NGDC table from 2010 [12]. The last, uncertain, min-to-min value, i.e. the average through cycle 24, is marked by an open square. At this last point error bars extend up to the average value derived by including the NASA predictions and down to the value found by including the IPS predictions beyond present.

The top panel of Figure 2 displays global temperature variations since 1850 through the deviations from average level 1961-1990.

Reliable global temperature series require that two conditions are met. Firstly, good thermometers are needed for the measurements. But secondly, there must be a fair global coverage such that localized climate variations like the North-Atlantic Oscillation (NAO), or the El Niño/La Niña in the Pacific would not affect the result too much. With these conditions it is hardly possible to extend reliable analyses further back than 1850, which is the year of the start of the temperature series developed jointly by Hadley UK Meteorological Centre and East Anglia University in England [13,14].

Presently, the series are extended up to October 2013 and comprise the combined land-surface/sea-surface global temperature series, HadCRUT-4gl [2], shown in the upper panel of Figure 2, which is used here for the analyses. For the discussions here it should be noted that following 
the steep rise between 1980 and 2000, the global average temperatures flatten out after year 2000. The extension of the temperatures beyond present shown by the dashed line represents the average of global temperatures from 2001 to 2013.

\section{Relations between Solar Activity and Global Temperature}

It should be recalled that solar activity-related changes in global temperatures must arrive after the activity changes. The former DMI analysis [3] examined the correlation between sunspots and global temperatures for the interval from 1850 to 1980 and derived a value of 3 years for the delay that provided optimum correlation. In Figure 2 the cycle-average global temperatures are presented by the squares and filled circles, respectively, for the min-tomin and max-to-max intervals shifted 3 years.

The averaging presented in Figure 2 over min-to-min or max-to-max solar cycle intervals delayed by 3 years include years beyond present for the last two points. In the summations a reference value equal to the mean value of global temperatures from 2001 to 2013 has been substituted for values beyond 2013. Error bars extending from the two points represent the results obtained with global temperatures beyond 2013 systematically defined $0.1^{\circ} \mathrm{C}$ higher or lower than the reference value.

In Figure 3 the individual cycle values of the sunspot number, $\mathrm{SSN}_{\mathrm{A}}$, averaged over either min-to-min or maxto-max intervals of the solar cycle (appr. 11 years) and the change in global temperatures, $\Delta \mathrm{T}_{\mathrm{A}}$, averaged over the same interval length but delayed by 3 years, are shown by filled squares and circles, respectively. This way of averaging reduces the scatter and makes it easier to se the persistent relation between sunspots and global tempera tures. The relation was found statistically in the former analysis [3] to be: $\Delta \mathrm{T}_{\mathrm{A}}=0.009( \pm 0.002) \cdot \mathrm{SSN}_{\mathrm{A}}-0.70^{\circ} \mathrm{C}$.

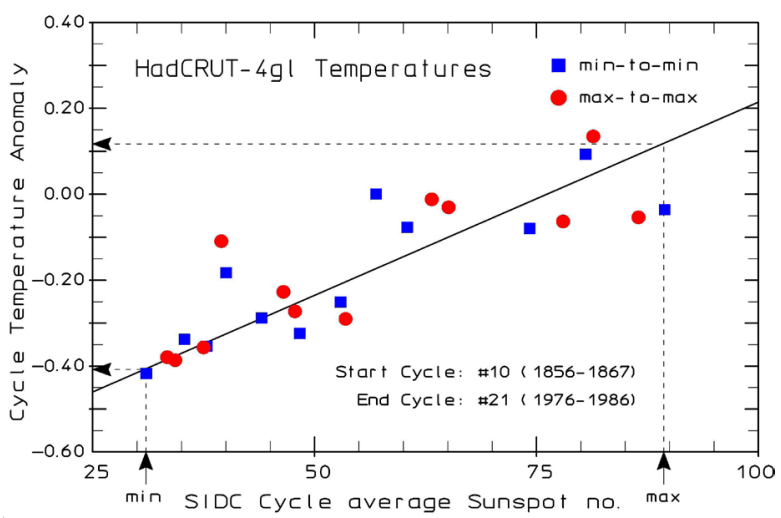

Figure 3. Average temperature anomaly (deviation from mean level 1961-1990) vs. average sunspot number through all min-to-min and max-to-max solar cycles through 1850 to 1980. Temperature intervals delayed by 3 years. The line has the slope $0.009^{\circ} \mathrm{C} / \mathrm{SSN}$.
The slope of the line in Figure 3 represents the relation: $\Delta \mathrm{T}_{\mathrm{A}} \sim 0.009 \cdot \mathrm{SSN}_{\mathrm{A}}$.

\section{Modified Temperatures}

With the relation presented in Figure 3 the cycle-average sunspot numbers can now be converted to average solar activity-related global temperature increases during the same interval length but delayed by 3 years. Thus, with the sunspot data presented in Figure 2 it is possible to calculate the values that the global temperatures would have taken without the solar activity-related contributions. Using the 2013 HadCRUT-4gl temperature series [2] as the temperature reference, the result is shown in Figure 4. There are, like in Figures 2 and 3, different signatures for values found by averaging through minto-min and max-to-max intervals of the solar cycles.

For the averaging of temperature values for the last two points in Figure 4, a reference value derived as the mean of global temperatures from 2001 to 2013 has been substituted for temperature values beyond present. The error bars extending from these two points correspond to temperatures systematically $0.1^{\circ} \mathrm{C}$ higher or lower than the reference value on top of the deviations caused by the range of predicted sunspot numbers through the final part of cycle 24 illustrated by the error bars on the last average sunspot point in Figure 2.

In Figure 4 the general level of the modified HadCRUT-4gl temperature anomalies has been displaced to provide a reference base line $\left(0.0^{\circ} \mathrm{C}\right)$ for the global temperatures corrected for the solar activity contributions calculated according to the relation provided in the former analysis [3]. It is seen that there are deviations of about $0.1^{\circ} \mathrm{C}$ to $0.2^{\circ} \mathrm{C}$ around this level at times between 1850 and 1980. However, from around 1985 a new development is seen. Now, the cycle-average global temperatures rise strongly to reach a value in 2010 almost $0.7^{\circ} \mathrm{C}$ above the base line. After 2010 the steady rise in the modified temperatures continues despite the actual

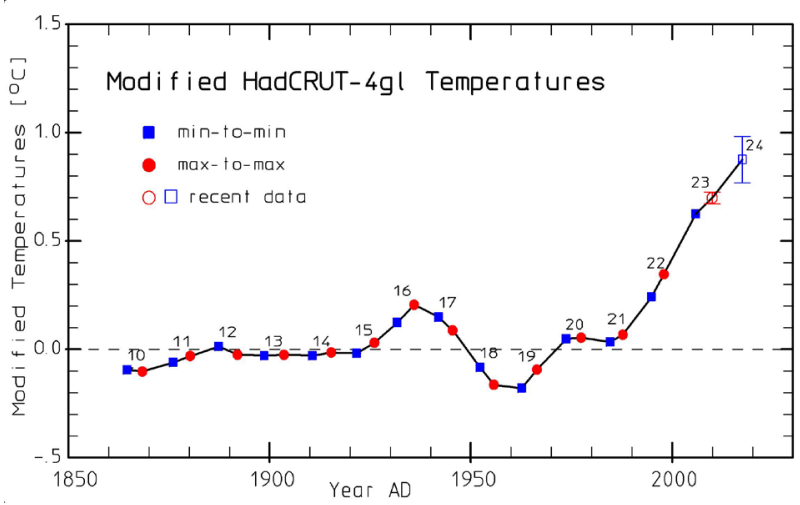

Figure 4. Cycle average global temperature anomalies (HadCRUT-4gl) corrected for contributions from solar activity. Solar cycle numbers are noted at the points. 
levelling-off in the observed temperatures.

\section{Discussions}

The purpose of the present communication is not to predict future temperatures but to disclose the effects of the declining solar activity. The development in the global temperatures corrected for the contributions from solar activity displays a steady rise with no indication of the levelling-off of the temperature rise after year 2000 that is seen in the original observed HadCRUT-4gl temperature data [2] shown in Figure 2.

The above presentation is based on using the global land/sea surface temperature series, HadCRUT-4gl. However, nearly the same numerical results and quite the same conclusions are reached when using the other standard temperature series [2], such as the global land surface temperatures, CruTEM-4gl, and the global sea-surface temperature series, HadSST-3gl, all updated in October 2013.

A further aspect is the possible changes in the sunspot data series supplied from Solar Influences Data Center [8]. A working group has in a preliminary conclusion suggested that the present international sunspot number, SSN, is overestimated by around 18\% after 1945 [15]. Calculations using the since 1945 reduced sunspot numbers have given almost the same slope $\left(0.009^{\circ} \mathrm{C} / \mathrm{SSN}\right)$ based on data from 1850 to 1980 - actually with a slightly smaller standard deviation. Furthermore, the temperature corrections implied in the construction of the modified HadCRUT-4gl temperatures displayed in Figure 4 are changed by insignificant amounts only by using the modified sunspot numbers.

\section{Conclusions}

The decaying solar activity makes the recently recorded global temperatures flatten out and thus disguises the real climate development. With a steady level of cycle-average solar activity the global temperatures would have shown a steady rise from 1980 to present (2013) in agreement with the increasing atmospheric concentrations of green-house gasses, primarily carbon dioxide and methane [16], and not the levelling-off actually observed since 2001.

The solar activity is now at the lowest level seen in the past 100 years and could not go much lower. Thus, the observed global temperatures may soon resume the steady rise observed from around 1980 to 2001. If solar activity starts increasing then the global temperatures may rise even steeper than that seen over the past three decades.

\section{REFERENCES}

[1] Intergovernmental Panel on Climate Change, IPCC 2013 Report. http://www.climatechange2013.org

[2] HadCRUT Global Temperature Data 2013. http://www.cru.uea.ac.uk/cru/data/temperature/

[3] P. Stauning, "Solar Activity-Climate Relations: A Different Approach," Journal of Atmospheric and Solar-Terrestrial Physics, Vol. 73, No. 13, 2011, pp. 1999-2012. http://dx.doi.org/10.1016/j.jastp.2011.06.011

[4] J. A. Eddy, “The Maunder Minimum,” Science, Vol. 192, No. 4245, 1976, pp. 1189-1202.

http://dx.doi.org/10.1126/science.192.4245.1189

[5] L. J. Gray, J. Beer, M. Geller, J. D. Haigh, M. Lockwood, K. Matthes, U. Cubasch, D. Fleitmann, G. Harrison, L. Hood, J. Luterbacher, G. A. Meehl, D. Shindell, B. van Geel and W. White, "Solar Influences on Climate," Reviews of Geophysics, Vol. 48, No. 4, 2010, pp. 1-53. http://dx.doi.org/10.1029/2009RG000282

[6] S. Engels and B. van Geel, "The Effects of Changing Solar Activity on Climate: Contributions from Palaeoclimatological Studies," Journal of Space Weather and Space Climate, Vol. 2, 2012, Article ID: A09. http://dx.doi.org/10.1051/swsc/2012009

[7] K. Mursula, P. Manoharan, D. Nandy, E. Tanskanen and P. Verronen, "Long-Term Solar Activity and Its Implications to the Heliosphere, Geomagnetic Activity, and the Earth's Climate," Journal of Space Weather and Space Climate, Vol. 3, 2013, Article ID: A21. http://dx.doi.org/10.1051/swsc/2013043

[8] Solar Influences Data Analysis Center (SIDC), Belgium, Sunspots, 2013.

http://sidc.oma.be/sunspot-data/, http://sidc.oma.be/silso

[9] D. H. Hathaway, R. M. Wilson and E. J. Reichmann, "The Shape of the Sunspot Cycle," Solar Physics, Vol. 151, No. 1, 1994, pp. 177-190. http://dx.doi.org/10.1007/BF00654090

[10] Australian IPS Radio and Space Service Predictions, 2013. http://www.ips.gov.au/Solar/1/6

[11] NASA Sunspot Predictions, 2013. http://solarscience.msfc.nasa.gov/predict.shtml

[12] NOAA-NGDC Solar Cycle Data, 2010. ftp://ftp.ngdc.noaa.gov/STP/SOLAR_DATA/SUNSPOT_ NUMBERS/maxmin.new

[13] P. Brohan, J. J. Kennedy, I. Harris, S. F. B. Tett and P. D. Jones, "Uncertainty Estimates in Regional and Global Observed Temperature Changes: A New Dataset from 1850," Journal of Geophysical Research: Atmospheres, Vol. 111, No. D12, 2006. http://dx.doi.org/10.1029/2005JD006548

[14] C. P. Morice, J. J. Kennedy, N. A. Rayner and P. D. Jones, "Quantifying Uncertainties in Global and Regional Temperature Change using an Ensemble of Observational Estimates: the HadCRUT4 Dataset,” Journal of Geophysical Research: Atmospheres, Vol. 117, No. D08, 2012. http://dx.doi.org/10.1029/2011JD017187

[15] E. Cliver, F. Clette and L. Svalgaard, "Central European Astrophysical Bulletin,” Vol. 37, 2013, p. 401.

[16] WMO Report on Greenhouse Gas Concentrations. http://www.wmo.int/pages/mediacentre/press_releases/pr _980_en.html 